\title{
A study of the tribological behavior of carbon-nanotube-reinforced ultrahigh molecular weight polyethylene composites
}

\author{
Z. Wei, ${ }^{1}$ Ya-Pu Zhao, ${ }^{1 *}$ S. L. Ruan, ${ }^{2}$ P. Gao ${ }^{3}$ and T. X. $\mathrm{Yu}^{2}$ \\ ${ }^{1}$ State Key Laboratory of Nonlinear Mechanics, Institute of Mechanics, Chinese Academy of Sciences, Beijing, 100080, PR China \\ 2 Dept of Mechanical Engineering, The Hong Kong University of Science and Technology, Clear Water Bay, Kowloon, Hong Kong, PR China \\ ${ }^{3}$ Dept of Chemical Engineering, The Hong Kong University of Science and Technology, Clear Water Bay, Kowloon, Hong Kong, PR China
}

Received 23 June 2005; Revised 18 October 2005; Accepted 19 October 2005

\begin{abstract}
The tribological properties of the high-strength and high-modulus ultrahigh molecular weight polyethylene (UHMWPE) film and the UHMWPE composites reinforced by multiwalled carbon nanotubes (MWCNT/UHMWPE) were investigated using a nanoindenter and atomic force microscope (AFM). The MWCNT/UHMWPE composites films exhibited not only high wear resistance but also a low friction coefficient compared to the pure UHMWPE films. We attribute the high wear resistance to the formation of the new microstructure in the composites due to the addition of MWCNTs. Copyright $\subset 2006$ John Wiley \& Sons, Ltd.
\end{abstract}

KEYWORDS: carbon nanotubes (CNTs); composite; wear; friction; microstructure

\section{INTRODUCTION}

Nanomaterials possess many special physical and chemical properties such as quantum-size effect, small-size effect, and surface and interface effects. Since the interaction between the polymer chains and the surface of the nanomaterials can evidently alter the chain kinetics in the region immediately surrounding the nanomaterials because of the presence of the interface, it is rational to anticipate that the use of nanomaterials as additives will provide a well-bonded interface that will enable polymer-based nanocomposites to exhibit high performance. ${ }^{1-5}$ As a matter of fact, even low filler-weight fractions provide an enormous interfacial area through which the bulk properties of the polymer can be altered, if the filler is well dispersed in the polymer matrix. Accordingly, it will be possible to develop novel, high-performance, polymer-based nanocomposites by the proper combination of a polymer matrix with nanometer reinforcement. The unusual electrical and mechanical properties of carbon nanotubes (CNTs) have motivated a flurry of interest to exploit their applications in advanced composite materials, particularly polymer composites, to improve the performance of the matrix or to achieve new properties. It has been reported that CNTs

*Correspondence to: Ya-Pu Zhao, State Key Laboratory of

Nonlinear Mechanics, Institute of Mechanics, Chinese Academy of

Sciences, Beijing, 100080, PR China.

E-mail: yzhao@lnm.imech.ac.cn

Contract/grant sponsor: Research Grant Council of Hong Kong;

Contract/grant number: HKUST 6244100P.

Contract/grant sponsor: National Natural Science Foundation of

China (NSFC); Contract/grant numbers: 10225209; 90305020.

Contract/grant sponsor: Chinese Academy of Sciences;

Contract/grant number: KJCX2-SW-L2. are extremely strong with a strength of tens of GPa and exceptionally stiff with Young's modulus in TPa range, yet remarkably flexible with the break strain larger than $5 \%{ }^{6,7}$ In addition, CNTs have high aspect ratios ( $>100)$. These properties make the use of CNTs as fillers in nanocomposites interesting. The use of CNTs in some matrixes significantly reduces the coefficient of friction and the wear rate of such nanocomposites. ${ }^{8-10}$ Here, the nanotubes were seen to act as a lubricant, which leads to the observed lower coefficient of friction and reduced wear rate.

In a pioneering work done by Ruan, Gao, Yang and $\mathrm{Yu},{ }^{11}$ experimental observations were reported of the drastically enhanced toughness in the ultrahigh molecular weight polyethylene (UHMWPE) films due to the addition of $1 \mathrm{wt} \%$ multiwalled carbon nanotubes (MWCNTs). Since MWCNTs are mechanically very strong and have a high aspect ratio, CNTs can be expected to significantly improve the tribological properties of UHMWPE-based composites. To the best of our knowledge, no study has been reported so far on the tribological properties of MWCNT/UHMWPE composites. As the dimensions of components and loads used continue to decrease (such as in microelectromechanical systems, MEMS), scratch/wear and mechanical properties at the micro to nanoscales become very important. ${ }^{12}$ So our interest was to study these materials at a small scale and on light loading. UHMWPE-based composites with different weight fractions of MWCNTs and different draw ratios (DRs) were prepared and their tribological properties were examined under ambient conditions. The cause of the favorable effects of MWCNTs on the tribological properties of UHMWPE/MWCNT composites has also been investigated. 


\section{EXPERIMENTAL}

\section{Preparation of MWCNT/UHMWPE composite films}

On the preparation of MWCNT/UHMWPE composite, many details have been given in the literature. ${ }^{11}$ Precursory UHMWPE fibers without/with $1 \mathrm{wt} \%$ and $5 \mathrm{wt} \%$ MWCNT prepared by the gel extrusion process were preheated for $30 \mathrm{~min}$ at $120^{\circ} \mathrm{C}$, pressed with a load of 20 tons for $30 \mathrm{~min}$ to get precursory films with a thickness $\sim 10 \mu \mathrm{m}$ and width $\sim 2 \mathrm{~mm}$, and then cooled to room temperature. The highly oriented fibers with a DR (length ratio) of 5, 11, 15, 20, 25, and 30 were prepared using MTS RT/ 5 with the oven in two steps (in the first step the precursor films were drawn to five times the length, $\mathrm{DR}=5$, at $120^{\circ} \mathrm{C}$, and in the second step, the above fibers were continued to be drawn to a predefined $\mathrm{DR}$ at $\left.130^{\circ} \mathrm{C}\right)$. After the fiber was drawn, it was put under a hot press again with a load of $\sim 25$ tons for $30 \mathrm{~min}$ at $120^{\circ} \mathrm{C}$, and then cooled to room temperature. Pressed fibers were put into an ethanol solution to clean them of impurities and for them to be closely contacted with the silicon wafer at the microscale. This sample was then ready for use in atomic force microscope (AFM)/nanoindenter tests directly.

\section{Friction and wear test}

Friction and wear tests were performed on a nanoindenter (TriboIndenter, Hysitron Incorporated, USA). The nanoindenter was equipped with a Berkovich diamond probe tip, a 3-sided pyramidal indenter. The total included angle of this tip was 142.3 degrees, with a half angle of 65.35 degrees. The tip radius was around $100 \mathrm{~nm}$. The nanoscratch test was used in order to investigate the resistance of the material with various CNT additions and DRs. This novel technique involved moving a sample while it is in contact with the diamond tip. The friction coefficient was determined from the ratio of the lateral force to the normal force. Therefore, the coefficient of friction indicates the resistance of the material to the tip penetration in a tangential direction. In this work, the normal force was kept as $75 \mu \mathrm{N}$ during the experiment. The scratch length was about $5 \mu \mathrm{m}$. The scratch velocity was $5 \mu \mathrm{m} / \mathrm{s}$.

The tip used for scratching can also be used for wear testing. The worn areas resulted from a tip scan from the top to the bottom over a square area. Using this type of testing, the durability of the materials can be compared. In this test, the topography scan was performed with a normal force of $2 \mu \mathrm{N}$, and the scale was $10 \times 10 \mu \mathrm{m}^{2}$, while the worn area was $5 \times 5 \mu \mathrm{m}^{2}$. Normal load in the wear was kept at $15 \mu \mathrm{N}$. Sliding speed was kept constant at $5 \mu \mathrm{m} / \mathrm{s}$. The number of wear cycles was 1,5 , or 12 . These experiments were conducted in air with $30-50 \%$ humidity and at room temperature $\left(22-25^{\circ} \mathrm{C}\right)$.

\section{RESULTS AND DISCUSSION}

\section{Nanoscratch tests}

The friction coefficient of the films was measured through the nanoscratch test. The normal load for the scratch test was fixed at $75 \mu \mathrm{N}$. The scratch length was set to be $5 \mu \mathrm{m}$. The normal force, lateral force, normal displacement, and lateral displacement during the scratch test were recorded with respect to time. The normal force was divided by the lateral force to calculate the friction coefficient of the films. Specimens with two DRs and three additions were used in this study.

The results of the friction tests for these specimens are summarized in Fig. 1. The MWCNT/UHMWPE composite films exhibit low friction coefficient in comparison with the pure UHMWPE films. The friction coefficient decreases with increasing weight fraction of CNTs in the composites.

\section{Nanowear test}

Figure 2 shows a typical microwear scar on the films. Variations of the depth of the wear scar can be obtained according to the cross section of the worn area.

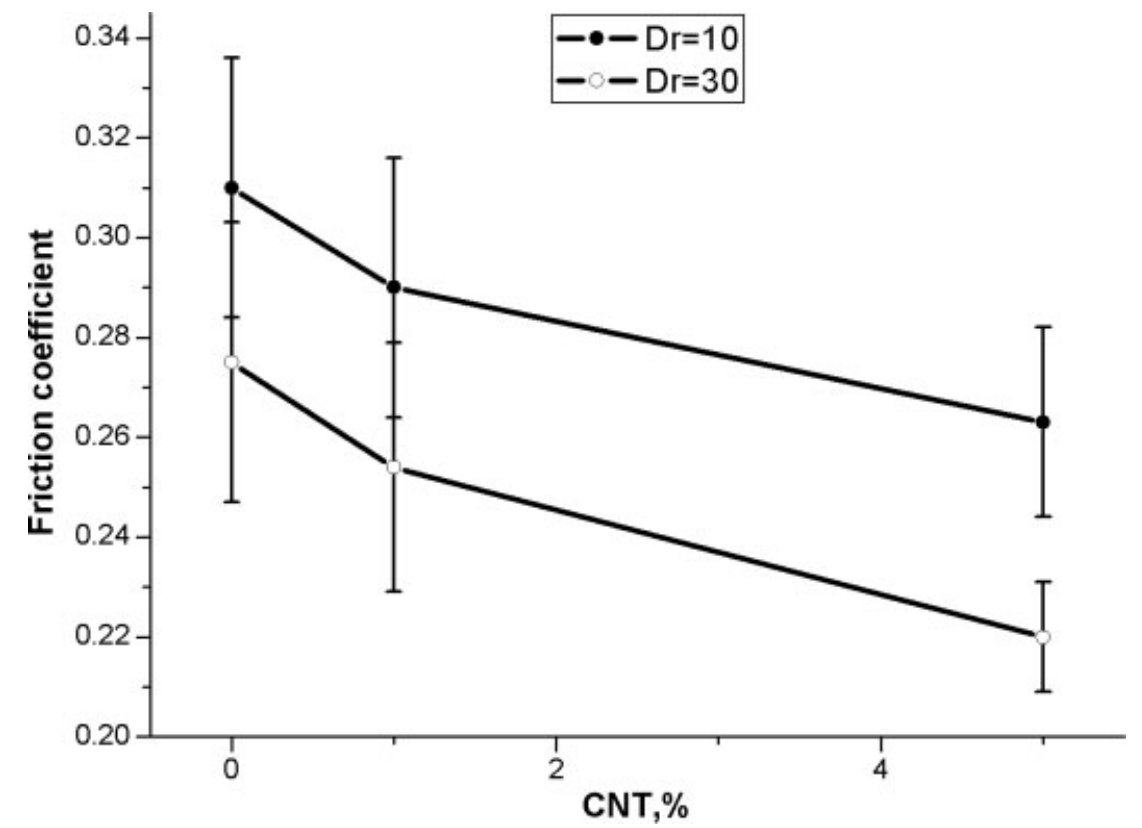

Figure 1. Variations of friction coefficient as a function of CNT addition in different draw ratio during scratch test. 


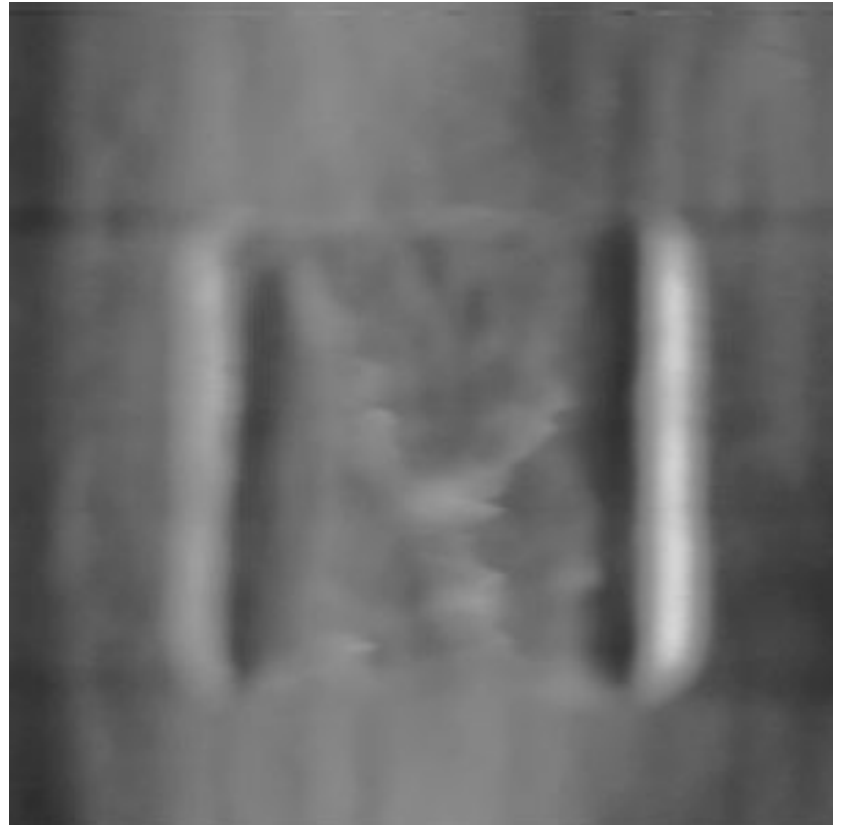

Figure 2. Example of an AFM image of the wear scar, the image size is $10 \mu \mathrm{m} \times 10 \mu \mathrm{m}$.

Since the heights at only a few points in the wear zone would not be sufficient to characterize the status of a wear test, a statistical processing was carried out in the wear zone. Here, for a convenient expression of wear, we define a mean wear depth as

$$
\bar{h}=\frac{1}{A} \int_{\sigma}\left|h(x, y)-h_{0}(x, y)\right| \mathrm{d} \sigma \quad \text { with } \quad A=\iint_{\sigma} \mathrm{d} x \mathrm{~d} y
$$

where $h_{0}$ is the height function of film before wear and $h$ is the height function after wear. The wear area was $5 \times 5 \mu \mathrm{m}^{2}$, and the topography measurement area was $10 \times 10 \mu \mathrm{m}^{2}$. The integral zone was $10 \times 10 \mu \mathrm{m}^{2}$. According to the abovedefined mean wear depth, the test data were processed and the results are shown in Fig. 3.

For the same material, the depth of the wear scar increases with increasing wear cycles. It is also seen that the depth of the wear scar decreases with increasing CNT addition regardless of DR. It is obvious that the MWCNT/UHMWPE composite films possess good wear resistance under such experimental conditions.

\section{DISCUSSIONS}

In order to investigate the enhancement mechanism of the composite, more tests were performed by AFM. Scratching is a powerful tool in AFM for characterizing mechanical properties of the samples on or near the surfaces. An important advantage of AFM over other instruments is that the same tool, the AFM cantilever, can be used for imaging immediately after scratch.

Many scratch tests were done on MWCNT/UHMWPE films and UHMWPE films. Figure 4 shows the images of scratch on MWCNT/UHMWPE and UHMWPE films. More material deposits were piled up near the scratches on the UHMWPE film than on the MWCNT/UHMWPE film. Moreover, on the surface of the MWCNT/UHMWPE film, a new microstructure could be observed, which is different from that on the UHMWPE film. This observation suggests that this microstructure is the interface between the CNTs and UHMWPE. Many strips dragged along the scratch also suggest that the microstructure has strong bonding between CNTs and UHMWPE. The microstructure results in the enhancement of the chain mobility of MWCNT/UHMWPE. ${ }^{11}$ We believe that it is this new microstructure that changed the properties of the pure UHMWPE film. A hypothesis we make here is that the formation of this new microstructure is due to the addition of CNTs. As CNTs have high strength and aspect ratio, the bonding between CNTs and UHMWPE is much stronger than those within UHMWPE itself. Therefore, the chain mobility of the MWCNT/UHMWPE composite is enhanced. Hence, the fracture cracks in the MWCNT/UHMWPE composite experience more difficulty in propagating. Therefore, the nanotube-reinforced composite has better wear resistance properties than UHMWPE.

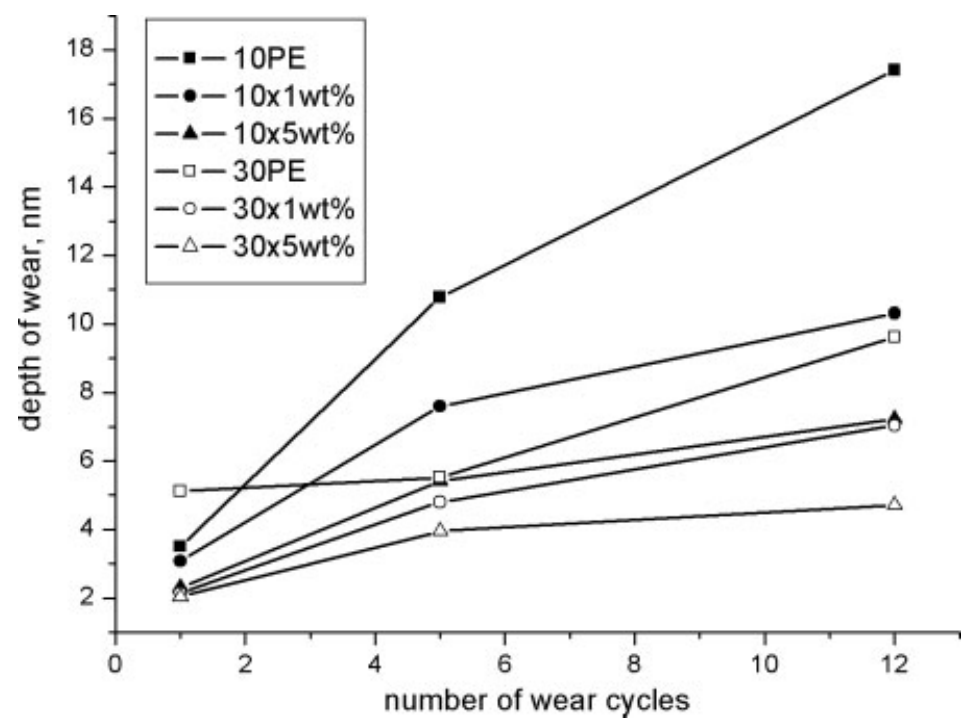

Figure 3. Variations of wear depth as functions of wear cycles with different CNT additions and draw ratios during wear test. 

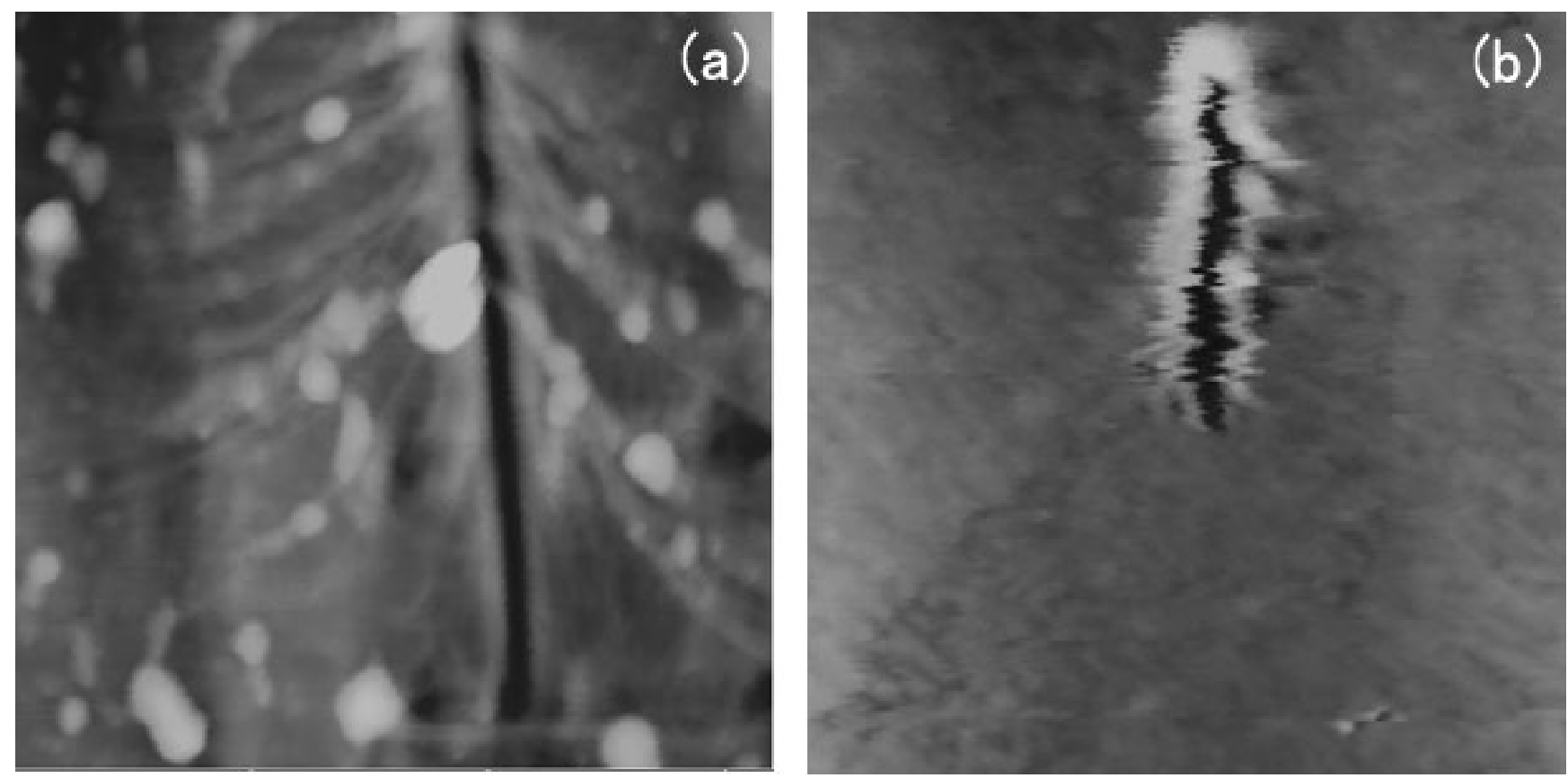

Figure 4. In situ AFM observations of a scratch, the image area is $2 \mu \mathrm{m} \times 2 \mu \mathrm{m}$. (a) MWCNT/UHMWPE film; (b) UHMWPE film.

As for the low friction coefficient of the MWCNT/ UHMWPE composite, some authors believe that it is caused by the self-lubrication of CNTs. ${ }^{10}$ We used the AFM tip to scratch the composite, but the subsequent examination of the used AFM tip by Raman spectroscopy did not support this explanation.

\section{CONCLUSIONS}

1. The MWCNT/UHMWPE composite films exhibit high wear resistance and low friction coefficient compared with pure UHMWPE films.

2. Because of the addition of MWCNTs, a new microstructure is formed. This new microstructure has strong interface bonding between MWCNTs and UHMWPE. Consequently, the MWCNT/UHMWPE composite films exhibit high wear resistance compared to the pure UHMWPE films.

\section{Acknowledgements}

This project was funded by the Research Grant Council of Hong Kong under an earmarked grant No. HKUST 6244100P, National Natural Science Foundation of China (NSFC) (grant No. 10225209 and No. 90305020), and key project from the Chinese Academy of Sciences (grant No. KJCX2-SW-L2). This work was done during the first author's visit to HKUST under a support from HKUST's VP-AA
Office via grant CMI03/04.EG01. These supports are gratefully acknowledged. The authors are also grateful for the opening funds from the Central Laboratory of Strength and Vibration at Tsinghua University and the Key Lab of Failure Mechanics (FML), Chinese Ministry of Education, Tsinghua University.

\section{REFERENCES}

1. Gao YJ, Sun R, Zhang ZJ, Xue QJ. Mater. Sci. Eng., A 2000; 286: 149.

2. Yoshida M, Deepak Kumar N, Prasad PN. J. Mater. Sci. 1997; 32: 4047.

3. Agag T, Koga T, Takeichi T. Polymer 2001; 42: 3399.

4. Pozdnyakov AO, Friedrich K, Kudryavtsev VV. J. Mater. Sci. Lett. 2001; 20: 2071.

5. Zhu ZK, Yang Y, Yin J, Qi ZN. J. Appl. Polym. Sci. 1999; 73: 2977.

6. Baughman RH, Zakhidov AA, de Heer WA. Science 2002; 297: 787.

7. Yu MF, Lourie O, Dyer MJ, Moloni K, Kelly TF, Ruoff RS. Science 2000; 287: 637.

8. Dong SR, Tu JP, Zhang XP. Mater. Sci. Eng., A 2001; 313: 83.

9. Chen WX, Tu JP, Wang LY, Gan HY, Xu ZD, Zhang XB. Carbon 2003; 41: 215.

10. Wang LY, Tu JP, Chen WX, Wang YC, Liu XK, Olk C, Cheng DH, Zhang XB. Wear 2003; 254: 1289.

11. Ruan SL, Gao P, Yang XG, Yu TX. Polymer 2003; 44: 5643.

12. Bhushan B. Handbook of Micro/Nanotribology 2nd edn, CRC Press: Boca Raton, Florida, 1999. 